\title{
Spectra and anisotropy of magnetic fluctuations in the Earth's magnetosheath: Cluster observations
}

\author{
O. Alexandrova ${ }^{1}$, C. Lacombe ${ }^{2}$, and A. Mangeney ${ }^{2}$ \\ ${ }^{1}$ University of Cologne, Institute of Geophysics and Meteorology, Albertus-Magnus-Platz 1, 50923 Cologne, Germany \\ ${ }^{2}$ LESIA, Observatoire de Paris, CNRS, UPMC, Université Paris Diderot, 5 place J. Janssen, 92190 Meudon, France
}

Received: 7 May 2008 - Revised: 11 September 2008 - Accepted: 29 September 2008 - Published: 17 November 2008

\begin{abstract}
We investigate the spectral shape, the anisotropy of the wave vector distributions and the anisotropy of the amplitudes of the magnetic fluctuations in the Earth's magnetosheath within a broad range of frequencies $\left[10^{-3}, 10\right] \mathrm{Hz}$ which corresponds to spatial scales from $\sim 10$ to $10^{5} \mathrm{~km}$. We present the first observations of a Kolmogorov-like inertial range of Alfvénic fluctuations $\delta B_{\perp}^{2} \sim f^{-5 / 3}$ in the magnetosheath flanks, below the ion cyclotron frequency $f_{c i}$. In the vicinity of $f_{c i}$, a spectral break is observed, like in solar wind turbulence. Above the break, the energy of compressive and Alfvénic fluctuations generally follows a power law with a spectral index between -3 and -2 . Concerning the anisotropy of the wave vector distribution, we observe a clear change in its nature in the vicinity of ion characteristic scales: if at MHD scales there is no evidence for a dominance of a slab $\left(k_{\|} \gg k_{\perp}\right)$ or 2-D $\left(k_{\perp} \gg k_{\|}\right)$turbulence, above the spectral break, $\left(f>f_{c i}, k c / \omega_{p i}>1\right)$ the 2-D turbulence dominates. This 2-D turbulence is observed in six selected one-hour intervals among which the average ion $\beta$ varies from 0.8 to 10 . It is observed for both the transverse and compressive magnetic fluctuations, independently on the presence of linearly unstable modes at low frequencies or Alfvén vortices at the spectral break. We then analyse the anisotropy of the magnetic fluctuations in a time dependent reference frame based on the field $\mathbf{B}$ and the flow velocity $\mathbf{V}$ directions. Within the range of the 2-D turbulence, at scales $[1,30] k c / \omega_{p i}$, and for any $\beta$ we find that the magnetic fluctuations at a given frequency in the plane perpendicular to $\mathbf{B}$ have more energy along the $\mathbf{B} \times \mathbf{V}$ direction. This non-gyrotropy of the fluctuations at a fixed frequency is consistent with gyrotropic fluctuations at a given wave vector, with $k_{\perp} \gg k_{\|}$, which suffer a different Doppler shift along and perpendicular to $\mathbf{V}$ in the plane perpendicular to $\mathbf{B}$.
\end{abstract}

Correspondence to: O. Alexandrova

(alex@geo.uni-koeln.de)
Keywords. Magnetospheric physics (Magnetosheath) Space plasma physics (Turbulence)

\section{Introduction}

In the space plasma turbulence, the presence of a mean magnetic field $\mathbf{B}$ gives rise to anisotropies with respect to the field direction (\| means parallel, and $\perp$ means perpendicular to B). There are anisotropies both in the intensities $\delta B^{2}$ of the magnetic fluctuations $\left(\delta B_{\perp}^{2} \neq \delta B_{\|}^{2}\right)$ and in the distribution of their wave vectors $\mathbf{k}\left(k_{\perp} \neq k_{\|}\right)$, i.e., the energy distribution of the turbulent fluctuations is anisotropic in $\mathbf{k}$-space.

To study the anisotropy of turbulent fluctuations in space plasma, we chose here the Earth's magnetosheath as a laboratory. Downstream of the bow shock, the solar wind plasma slows down, and the plasma density, temperature and magnetic field increase in comparison with the solar wind plasma. The magnetosheath boundaries (bow shock and magnetopause) introduce an important ion temperature anisotropy $T_{\perp}>T_{\|}$, and therefore linearly unstable waves, such as Alfvén Ion Cyclotron (AIC) and mirror modes, are present (see the reviews by Schwartz et al., 1996; Lucek et al., 2005; Alexandrova, 2008). In the vicinity of the bowshock, an $f^{-1}$ power law spectrum is observed at frequencies below the ion cyclotron frequency, $f<f_{c i}$, (Czaykowska et al., 2001). The power law spectra $\sim f^{-5 / 3}$, typical of the solar wind inertial range at $f<f_{c i}$, have not been observed in the magnetosheath. However, as in the solar wind, the energy of the magnetic fluctuations follows a power law close to $\sim f^{-3}$ at frequencies $f>f_{c i}$ (Rezeau et al., 1999; Czaykowska et al., 2001).

The question of the anisotropy of wave vectors in the magnetosheath has been mostly addressed for dominant frequencies (spectral peaks) in the turbulent spectrum, below $f_{c i}$,

Published by Copernicus Publications on behalf of the European Geosciences Union. 
where linearly unstable modes are expected (Alexandrova et al., 2004; Schäfer et al., 2005; Narita et al., 2006; Narita and Glassmeier, 2006; Constantinescu et al., 2007). Instead, we are interested in permanent fluctuations in the magnetosheath (and not in spectral peaks) which cover a very broad range of frequencies (more than 5 decades), from frequencies well below $f_{c i}$ to frequencies much higher than $f_{c i}$.

These permanent fluctuations within the frequency range $[0.35,12.5] \mathrm{Hz}$, above $f_{c i}$, and for one decade of scale lengths around Cluster separations $(\sim 100 \mathrm{~km})$, have been studied by Sahraoui et al. (2006) using the $k$-filtering technique. For a relatively short time interval in the inner magnetosheath (close to the magnetopause) and for a proton beta $\beta_{i} \sim 5$, the authors show that the wave-vectors of the fluctuations are mostly perpendicular to the mean magnetic field $\mathbf{B}, k_{\perp} \gg k_{\|}$, and that their frequency $\omega_{0}$ in the plasma frame is zero. In the plane perpendicular to $\mathbf{B}$, the $\mathbf{k}$-distribution is non-gyrotropic, more intense and with a well-defined power law $k^{-8 / 3}$ in a direction along the flow velocity $\mathbf{V}$ which was perpendicular to both $\mathbf{B}$ and the normal to the magnetopause for this particular case. The presence of linearly unstable large scale mirror mode during the considered time interval makes the authors conclude that the small scale fluctuations with the observed dispersion properties $k_{\perp} \gg k_{\|}$and $\omega_{0}=0$ result from a non-linear cascade of mirror modes.

At higher frequencies, $\sim\left[10,10^{3}\right] \mathrm{Hz}$, between about the lower hybrid frequency $f_{l h}$ and 10 times the electron cyclotron frequencie $f_{c e}$, the permanent fluctuations observed in the magnetosheath, during four intervals of several hours, have been studied by Mangeney et al. (2006) and Lacombe et al. (2006). The corresponding spatial scales, $\sim[0.1,10] \mathrm{km}$ $\simeq[0.3,30] k c / \omega_{p e}\left(c / \omega_{p e}\right.$ being the electron inertial length $)$, are much smaller than the Cluster separations, and so only the one-spacecraft technique could be used to analyze the anisotropy of wave vector distributions.

Magnetic fluctuations with $k_{\|} \gg k_{\perp}$, usually called "slab turbulence", have rapid variations of the correlation function along the field and weak dependence upon the perpendicular coordinates. For the fluctuations with $k_{\perp} \gg k_{\|}$, called "2$\mathrm{D}$ turbulence", the correlation function varies rapidly in the perpendicular plane, and there is no dependence along the field direction. So, measurements along different directions with respect to the mean field can give the information on the wave vector anisotropy. Under the assumption of convected turbulent fluctuations through the spacecraft (i.e., the phase velocity $v_{\phi}$ of the fluctuations is small with respect to the flow velocity), these measurements are possible with one spacecraft thanks to the variation of the mean magnetic field $\mathbf{B}$ direction with respect to the bulk flow $\mathbf{V}$. While $\mathbf{V} \| \mathbf{B}$, the spacecraft resolve fluctuations with $\mathbf{k} \| \mathbf{B}$, when $\mathbf{V} \perp \mathbf{B}$, the fluctuations with $\mathbf{k} \perp \mathbf{B}$ are measured.

This idea was already used in the solar wind for studying the wave vector anisotropies of the Alfvénic fluctuations in the inertial range (Matthaeus et al., 1990; Bieber et al., 1996; Saur and Bieber, 1999). The authors suppose that the observed turbulence is a linear superposition of two uncorrelated components, slab and 2-D, and both components have a power law energy distribution with the same spectral index $s, \delta B_{\perp}^{2}\left(k_{\|}\right) \sim A_{1} k_{\|}^{-s}$ and $\delta B_{\perp}^{2}\left(k_{\perp}\right) \sim A_{2} k_{\perp}^{-s}$, where $A_{1}$ and $A_{2}$ are the amplitudes of slab and 2-D turbulent components, respectively. Bieber et al. (1996) propose two independent observational tests for distinguishing the slab component from the 2-D component.

The first test is based on the anisotropy of the power spectral density (PSD) of the magnetic fluctuations in the plane perpendicular to $\mathbf{B}$, i.e., on the non-gyrotropy of the PSD at a given frequency in the spacecraft frame: in the case of a slab turbulence with $\mathbf{k} \| \mathbf{B}$, all the wave vectors suffer the same Doppler shift depending on the angle between $\mathbf{k}$ and $\mathbf{V}$, and if the spectral power is gyrotropic in the plasma frame, it will remain gyrotropic in the spacecraft frame; in the case of a 2-D turbulence, with $\mathbf{k} \perp \mathbf{B}$, if the PSD is gyrotropic in the plasma frame, it will be non-gyrotropic in the spacecraft frame because the Dopler shift will be different if $\mathbf{k}$ is perpendicular to $\mathbf{V}$ and if $\mathbf{k}$ has a component along $\mathbf{V}$.

The second test reveals the dependence of the PSD at a fixed frequency on the angle between the plasma flow and the mean field $\Theta_{B V}$ (defined between 0 and 90 degrees): For a PSD decreasing with $k$ (like a power-law, for example), in the case of the slab turbulence, the PSD for a given frequency will be more intense for $\Theta_{B V}=0^{\circ}$, and therefore, the PSD decreases while $\Theta_{B V}$ increases; for the 2-D turbulence the PSD will be more intense for $\Theta_{B V}=90^{\circ}$, and so it increases with $\Theta_{B V}$. Using these tests, Bieber et al. (1996) have shown that the subrange $\left[10^{-3}, 10^{-2}\right] \mathrm{Hz}$ in the inertial range of the slow solar wind is dominated by a 2-D turbulence; however, a small percentage of a slab component is present.

Mangeney et al. (2006) proposed a model of anisotropic wave vector distribution without any assumption on the independence of the two turbulence components. In their gyrotropic model, the wave vector can be oblique with respect to the $\|$ and $\perp$ directions. The authors introduce a cone aperture of the angle $\theta_{k B}$ between $\mathbf{k}$ and $\mathbf{B}$, as a free parameter of the model. They assume a power law distribution of the total energy of the fluctuations $\sim k^{-s}$, with $s$ independent on $\theta_{k B}$. For a given $k$, the turbulent spectrum is modeled by one of the two typical angular distribution $\sim \cos \left(\theta_{k B}\right)^{\mu}$ for $\mathbf{k}$ nearly parallel to $\mathbf{B}$ and $\sim \sin \left(\theta_{k B}\right)^{\mu}$ for $\mathbf{k}$ nearly perpendicular to B. For these two distributions, the cone aperture of $\theta_{k B}$ is about $20^{\circ}$ for $\mu=10$ and $7^{\circ}$ for $\mu=100$. The angle $\theta_{k B}$ can be easily represented through $\Theta_{B V}$ and so the model can be tested with one-spacecraft measurements.

An advantage of the magnetosheath with respect to the solar wind in ecliptical plane is that the angle $\Theta_{B V}$ covers the range from $0^{\circ}$ to $90^{\circ}$ within rather short time periods (one hour, or so) while other plasma conditions remain roughly the same. A comparison of the model described above with the observations of the total PSD of the magnetic fluctuations within the magnetosheath flanks, at frequencies between $f_{l h}$ and $10 f_{c e}$, shows that these fluctuations have 
a strongly anisotropic distribution of $\mathbf{k}$, with $\theta_{k B}=(90 \pm 7)^{\circ}$ (Mangeney et al., 2006). Actually, this model (as well as the tests of Bieber et al., 1996) is valid not only for a power law energy distribution in $k$, but for any monotone dependence where the energy decreases with increasing $k$.

Mangeney et al. (2006) have also shown that the variations of $\delta B^{2}$ with $\Theta_{B V}$ for a given frequency was not consistent with the presence of waves with a non-negligible phase velocity $v_{\phi}$. In other words, if the observed turbulent fluctuations are a superposition of waves, their $v_{\phi}$ has to be much smaller than the flow velocity for any wave number $k$. This is consistent with the assumption that the wave frequency $\omega_{0}$ is vanishing: the fluctuations are due to magnetic structures frozen in the plasma frame. These results have been obtained in the magnetosheath flanks for $f>10 \mathrm{~Hz}$, at electron spatial scales $\sim[0.3,30] k c / \omega_{p e}$.

In this paper we extend the study of Mangeney et al. (2006) to frequencies below $10 \mathrm{~Hz}$, for the same time periods in the magnetosheath flanks. As a result, we will cover the largest possible scale range, from electron $(\sim 1 \mathrm{~km})$ to MHD scales $\left(\sim 10^{5} \mathrm{~km}\right)$. At variance with the previous study, we analyse the spectral shapes and anisotropies for parallel ( $\sim$ compressive) $\delta B_{\|}$and for transverse ( $\sim$ Alfvénic) $\delta B_{\perp}$ fluctuations independently. For Alfvénic fluctuations $\delta B_{\perp}$ we perform the first test of Bieber et al. (1996), i.e., we analyze the gyrotropy of the PSD of the magnetic fluctuations in the plane perpendicular to $\mathbf{B}$, at a given frequency, as a function of $\Theta_{B V}$.

\section{Data and methods of analysis}

For our study we use high resolution (22 vectors per second) magnetic field waveforms measured by the FGM instrument (Balogh et al., 2001). Four seconds averages of the PSD of the magnetic fluctuations at 27 logarithmically spaced frequencies, between $8 \mathrm{~Hz}$ and $4 \mathrm{kHz}$, are measured by the STAFF Spectrum Analyser (SA) (Cornilleau-Wehrlin et al., 1997). Plasma parameters with a time resolution of $4 \mathrm{~s}$ are determined from HIA/CIS measurements (Rème et al., 2001).

\subsection{Magnetic spectra and decomposition in $\delta B_{\perp}$ and $\delta B_{\|}$}

High resolution FGM measurements allow to resolve turbulent spectra up to $\sim 10 \mathrm{~Hz}$. Similar to Alexandrova et al. (2006), we calculate the spectra of the magnetic fluctuations in the GSE directions X, Y and Z, using the Morlet wavelet transform. The total power spectral density (PSD) is $\delta B^{2}(f)=\sum_{j=X, Y, Z} \delta B_{j}^{2}(f)$. The PSD of the compressive fluctuations $\delta B_{\|}^{2}(f)$ is approximated by the PSD of the modulus of the magnetic field. This is a good approximation when $\delta B^{2} \ll B_{0}^{2}$, where $B_{0}$ is the modulus of the magnetic field at the largest scale of the analysed data set. The PSD of the transverse fluctuations is therefore

$\delta B_{\perp}^{2}(f)=\delta B^{2}(f)-\delta B_{\|}^{2}(f)$.

This approach, based on wavelet decomposition, allows the separation of $\delta B_{\perp}$ and $\delta B_{\|}$with respect to a local mean field, i.e. to the field averaged on a neighbouring scale larger than the scale of the fluctuations. The lower frequency limit of this approach is a scale where the ordering $\delta B^{2} \ll B_{0}^{2}$ is no longer satisfied.

The STAFF-SA instrument measures the spectral matrix $\left\langle\delta B_{i}(f) \delta B_{j}(f)\right\rangle$ at higher frequencies. Because of a recently detected error about the axes directions in the spin plane (O. Santolik, private communication, 2008) we cannot separate parallel and perpendicular spectra at the STAFF-SA frequencies; however we present here the total PSD, the trace of the spectral matrix.

\subsection{Anisotropy of the $\mathbf{k}$ distribution}

The motion of the plasma with respect to a probe allows a 1$\mathrm{D}$ analysis of the wave vector distribution along the direction of $\mathbf{V}$, as was discussed in Sect. 1. The 3-D wave vector power spectrum $I(\mathbf{k}) \equiv I\left(k, \theta_{k B}, \varphi_{k}\right)$ depends on the wave number $k$, on the angle $\theta_{k B}$ between $\mathbf{k}$ and $\mathbf{B}$, and on the azimuth $\varphi_{k}$ of $\mathbf{k}$ in the plane perpendicular to $\mathbf{B}$. If $\omega_{0}$ is the frequency of a wave in the plasma rest frame ( $\omega_{0}$ and $\omega$ are assumed to be positive), the Doppler shifted frequency $f=\omega / 2 \pi$ in the spacecraft frame is given by

$\omega=\left|\omega_{0}+\mathbf{k} \cdot \mathbf{V}\right|$.

The trace of the power spectral density at this frequency is

$\delta B^{2}(\omega)=A \int I(\mathbf{k}) \delta\left(\omega-\left|\omega_{0}+\mathbf{k} \cdot \mathbf{V}\right|\right) d \mathbf{k}$

i.e. the sum of the contributions with different $\mathbf{k}$. $A$ is a normalisation factor and $\delta$ the Dirac function.

The angle $\theta_{k B}$ can be considered as depending on the angle $\theta_{k V}$ between $\mathbf{k}$ and $\mathbf{V}$, the angle appearing in the Doppler shift frequency, and on the angle $\Theta_{B V}$ between $\mathbf{B}$ and $\mathbf{V}$ (see Eq. 2 of Mangeney et al., 2006). Thus, the variations of $\delta B^{2}$ with $\Theta_{B V}$ for a given $\omega$ will give information about $I(\mathbf{k})$. As was discussed in Sect. 1, $\delta B^{2}$ increases with increasing $\Theta_{B V}$ when the fluctuations have $k_{\perp} \gg k_{\|}$(2-D turbulence) and it decreases for a slab turbulence with $k_{\|} \gg k_{\perp}$ (see Fig. 6 of Mangeney et al., 2006). As a consequence, in the case of 2D turbulence, the spectrum of the fluctuations will be higher for large angles $\Theta_{B V}$ than for small ones, and vice-versa for the slab geometry.

\section{$2.3 \delta \mathbf{B}$-anisotropy in the $\mathbf{B V}$-frame}

To study the distribution of the $\operatorname{PSD} \delta B_{\perp}^{2}(f)$ in the plane perpendicular to $\mathbf{B}$, i.e. the gyrotropy of the magnetic fluctuations, taking into account the direction of the flow velocity $\mathbf{V}$, 


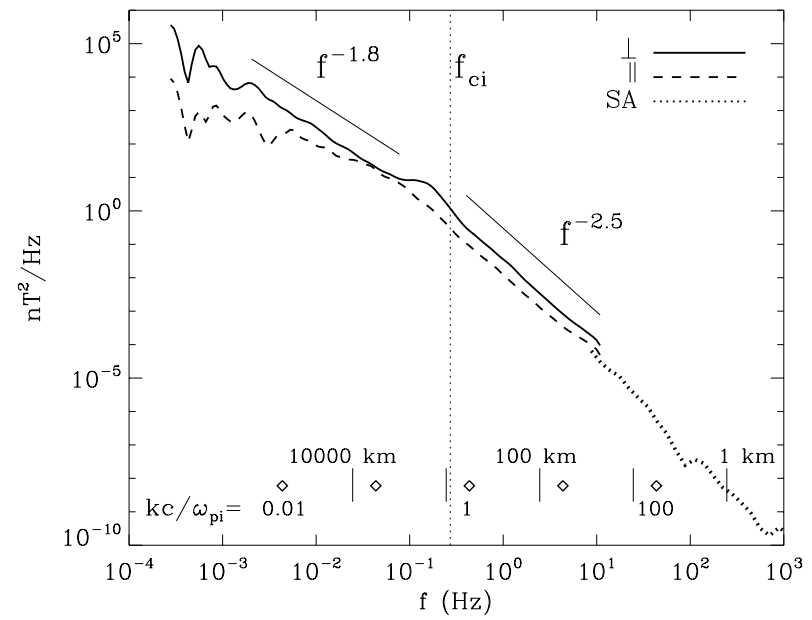

Fig. 1. FGM and STAFF-SA/Cluster data on 19 December 2001, 02:00-04:00 UT. Average spectra of the magnetic fluctuations, calculated using the Morlet wavelet transform of the FGM data $(f<10 \mathrm{~Hz})$. Solid line: for the transverse fluctuations $\delta B_{\perp}$. Dashed line: for the compressive fluctuations $\delta B_{\|}$. Dotted line: the total power spectral density, STAFF-SA data $(f>8 \mathrm{~Hz})$. The diamonds give the scales $k c / \omega_{p i} \simeq k r_{g i} \simeq 0.01$ to 100 . The vertical dotted line gives the average proton cyclotron frequency $f_{c i}$. The shapes of the power laws $f^{-1.8}$ and $f^{-2.5}$ are shown.

we shall consider the following reference frame $(\mathbf{b}, \mathbf{b v}, \mathbf{b b v})$ : $\mathbf{b}$ is the direction of the $\mathbf{B}$ field, $\mathbf{b v}$ the direction of $\mathbf{B} \times \mathbf{V}$ and $\mathbf{b b v}$ the direction of $\mathbf{B} \times(\mathbf{B} \times \mathbf{V})$.

The definition of this frame depends on the considered scale (frequency). A local reference frame (defined on a neighbouring scale larger than the scale of the fluctuations) is defined for frequencies below the spacecraft spin frequency $f_{\text {spin }}=0.25 \mathrm{~Hz}$ which limits the plasma moments time resolution to $4 \mathrm{~s}$. Then, for any frequency $f>f_{\text {spin }}$ we use the frame (b, bv, bbv) redefined every $4 \mathrm{~s}$.

In this frame, we only consider the frequencies below $10 \mathrm{~Hz}$, i.e., the FGM data (the STAFF-SA data cannot be used because of the error that has to be corrected in the whole data set). We project the wavelet transform of $B_{X}, B_{Y}$ and $B_{Z}$ on the $\mathbf{b}, \mathbf{b v}, \mathbf{b b v}$ directions and we calculate the squares of these projections $\delta B_{b}^{2}(f, t), \delta B_{b v}^{2}(f, t)$ and $\delta B_{b b v}^{2}(f, t)$ which are the diagonal terms of the spectral matrix in this new frame.

\section{3 k-distribution of $\delta B_{\perp}$ and $\delta B_{\|}$}

\subsection{A case study with $\beta_{i} \simeq 1$}

We consider an interval on 19 December 2001, from 02:00 to 04:00 UT. For this interval, the mean plasma parameters are the following: the magnetic field $B=(18 \pm 2) \mathrm{nT}$, the ion plasma density $N_{i}=(7 \pm 1) \mathrm{cm}^{-3}$, the ion temperature
$T_{i}=(120 \pm 15) \mathrm{eV}$ (with $\left.T_{i \perp} / T_{i \|}=1.7\right)$, the ion plasma beta $\beta_{i}=(1.1 \pm 0.4)$, the ion inertial length $c / \omega_{p i}=(90 \pm 5) \mathrm{km}$ and the ion Larmor radius $r_{g i}=(65 \pm 15) \mathrm{km}$. The electrons are isotropic and their temperature is small with respect to the ion temperature, $T_{e} \simeq 20 \mathrm{eV}$. The average upstream bow shock angle $\theta_{B N}$ calculated with the ACE data is about $70^{\circ}$ (Lacombe et al., 2006).

Figure 1 displays the average spectra of the FGM data for the transverse fluctuations (solid lines) and for the compressive fluctuations (dashed lines). The total PSD of the STAFFSA data is the dotted line above $8 \mathrm{~Hz}$. The total covered frequency range is more than six decades, from $3 \times 10^{-4} \mathrm{~Hz}$ to $10^{3} \mathrm{~Hz}$. The small vertical bars just above the abscissae-axis indicate the scales from $\lambda=10^{4} \mathrm{~km}$ to $1 \mathrm{~km}$ corresponding to the Doppler shift $f=V / \lambda$ for $\theta_{k V}=0^{\circ}$ and for the average velocity $V=(246 \pm 25) \mathrm{km} / \mathrm{s}$. The diamonds above the abscissae indicate the scales $k c / \omega_{p i} \simeq k r_{g i} \simeq 0.01$ to 100 , corresponding to the frequency $f=k V / 2 \pi$. Precisely, $k c / \omega_{p i}=1$ appears in the spectrum at $f=(0.44 \pm 0.05) \mathrm{Hz}$ and $k r_{g i}=1$ appears at $f=(0.63 \pm 0.11) \mathrm{Hz}$.

We see in Fig. 1 that, in the FGM frequency range, $\delta B_{\perp}^{2}(f)$ is everywhere larger than $\delta B_{\|}^{2}(f)$, except around $f \sim 5 \times 10^{-2} \mathrm{~Hz}$ where $\delta B_{\perp}^{2} \sim \delta B_{\|}^{2}$, and where the compressive fluctuations display a spectral break. The spectrum of $\delta B_{\perp}$ displays a bump and a break around $0.2 \mathrm{~Hz}$, that can be a signature of Alfvén vortices (Alexandrova et al., 2006). Below the bump, $\delta B_{\perp}^{2}(f) \sim f^{-1.8}$, a power law with an exponent close to the Kolmogorov's one $-5 / 3$ (in Sect. 5 we will analyse spectral shapes in more details). Above the bump, for $k c / \omega_{p i}>0.2, \delta B_{\perp}^{2}(f)$ and $\delta B_{\|}^{2}(f)$ follow a similar power law $\sim f^{-2.5}$. This power law extends on the STAFF-SA frequency range up to $k c / \omega_{p i} \simeq 50\left(k c / \omega_{p e} \simeq 1.2\right)$. It is quite possible that, above these scales, the dissipation of the electromagnetic turbulence starts. However, around $f \simeq 100 \mathrm{~Hz}$, there is another spectral bump, which is due to whistler waves, identified by their right-handed polarisation. The question of the turbulence dissipation is out of scope of the present paper and will be analysed in details in the future.

Now, we consider the anisotropy of the distribution of the wave vectors. Figure 2 shows the dependence of $\delta B_{\perp}^{2}$ (left column) and $\delta B_{\|}^{2}$ (right column) on the angle $\Theta_{B V}$ at different frequencies. The thick solid curves give the median values for bins $5^{\circ}$ wide. The upper panels of Fig. 2 correspond to $f=3 \mathrm{~Hz}\left(k c / \omega_{p i} \simeq 7\right)$. The observed increase of $\delta B_{\perp}^{2}$ and $\delta B_{\|}^{2}$ with $\Theta_{B V}$ can be produced only by fluctuations with $k_{\perp} \gg k_{\|}$, with phase velocities $v_{\phi}$ negligible with respect to the plasma bulk velocity, and with decreasing intensity of the fluctuations with increasing $k$ (as was discussed in Sects. 1 and 2.2).

At larger scales (lower frequencies) we observe the same tendency for $f>0.3 \mathrm{~Hz}$. The middle panels of Fig. 2 display $\delta B_{\perp}^{2}$ and $\delta B_{\|}^{2}$ as functions of $\Theta_{B V}$ for $f=0.52 \mathrm{~Hz}$ $\left(k c / \omega_{p i} \simeq 1.2\right)$ : we still observe here a clear increase of $\delta B_{\perp}^{2}$ and $\delta B_{\|}^{2}$ with $\Theta_{B V}$. The lower panels of Fig. 2 display $\delta B_{\perp}^{2}$ 

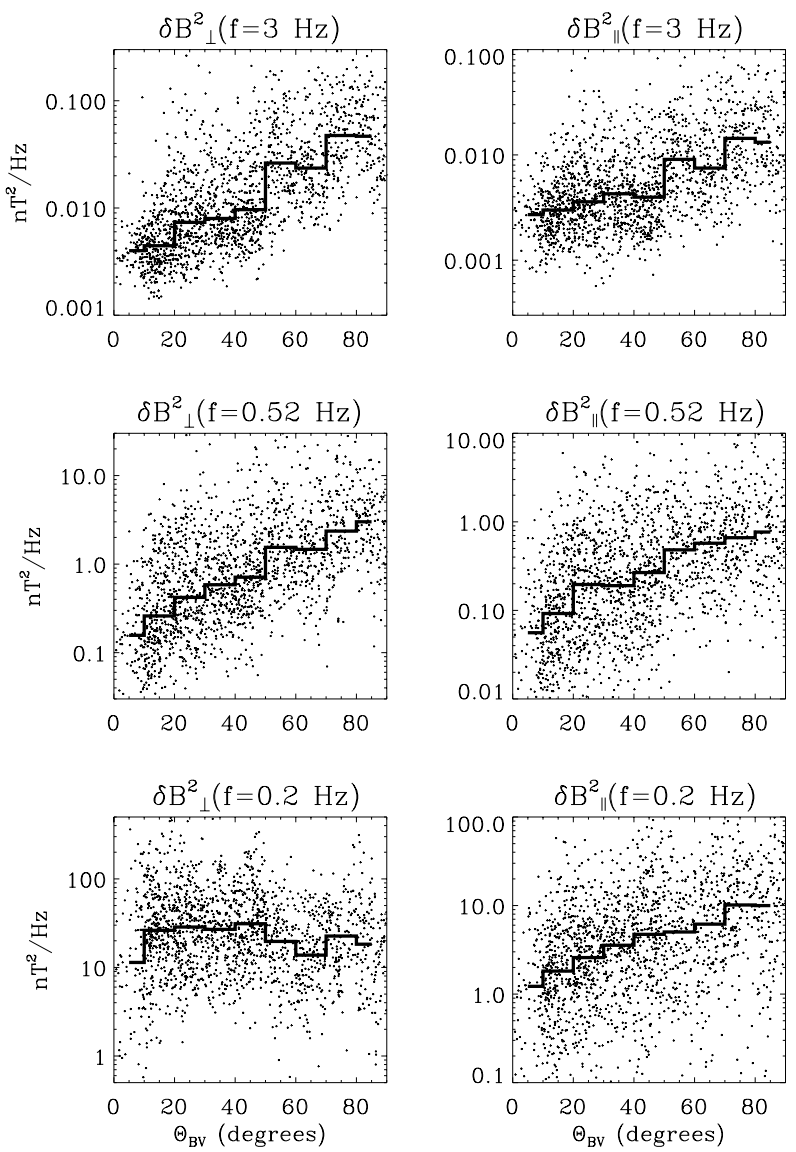

Fig. 2. FGM/Cluster data on 19 December 2001, 02:00-04:00 UT. Upper panels: Scatter plots of the power spectral density of the magnetic fluctuations at $f=3 \mathrm{~Hz}$ as a function of the angle between the local mean field and velocity, $\Theta_{B V}$. The distributions of the energy of Alfvénic fluctuations $\delta B_{\perp}^{2}$ is shown in the left panel, $\delta B_{\|}^{2}$ is shown in the right panel. Middle and lower panels have the same format, but here the frequencies are respectively $0.52 \mathrm{~Hz}$ and $0.2 \mathrm{~Hz}$. In all panels, the thick lines give the median value for bins $5^{\circ}$ wide.

and $\delta B_{\|}^{2}$ at $0.2 \mathrm{~Hz}\left(k c / \omega_{p i} \simeq 0.5\right)$, just at the spectral bump of $\delta B_{\perp}^{2}$ (see Fig. 1). $\delta B_{\|}^{2}$ still increases with $\Theta_{B V}$ (in spite of a large dispersion of the data points around the median), while $\delta B_{\perp}^{2}$ has a flat distribution with $\Theta_{B V}$. This can be due to several reasons: (i) $I(k)$ is no longer a decreasing function with $k$, (ii) $I\left(\theta_{k B}\right)$ is more isotropic in the spectral bump and/or (iii) the fluctuations are not frozen in plasma at this scale. This spectral bump, as we have already mentioned, can be the signature of Alfvén vortices with $k_{\perp} \gg k_{\|}$, propagating slowly in the plasma frame. It can be also the signature of propagating AIC waves with $k_{\|} \gg k_{\perp}$, which are unstable for the observed plasma conditions (Mangeney et al., 2006; Samsonov et al., 2007). However, as explained in Sects. 1 and 2.2, the energy of fluctuations with $k_{\|} \gg k_{\perp}$ would decrease with increasing $\Theta_{B V}$ at a given frequency, while in our case $\delta B_{\perp}^{2}$ seems to be independent on $\Theta_{B V}$.
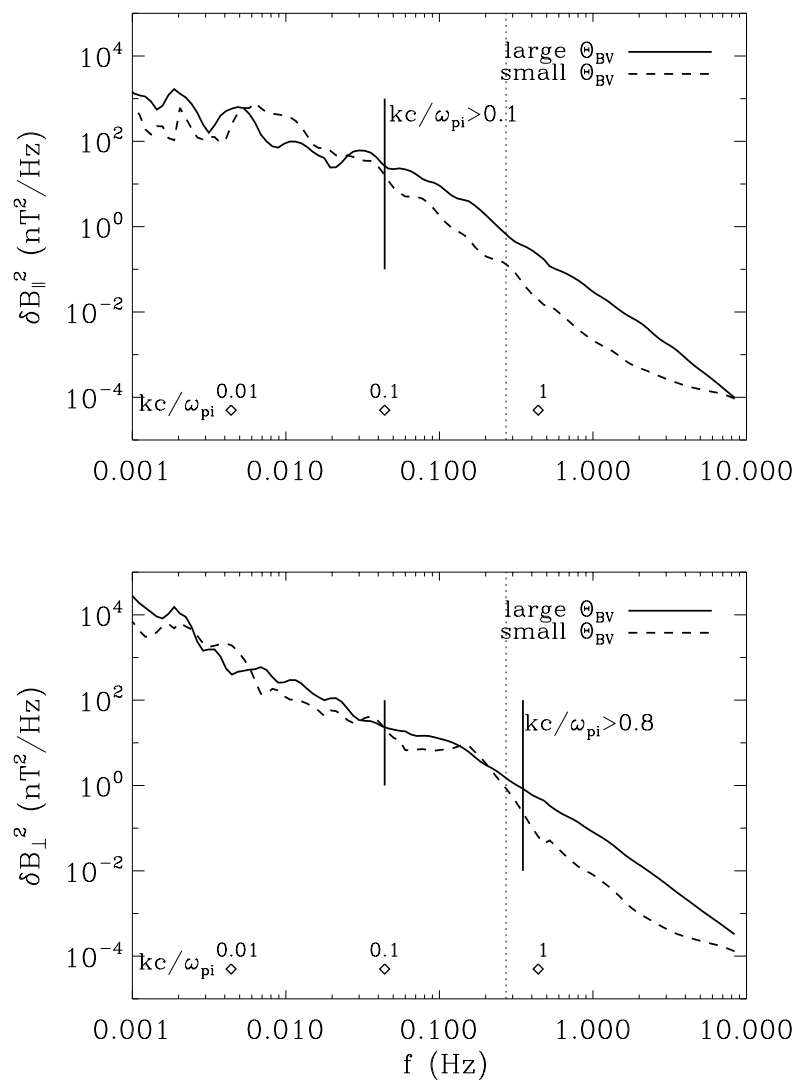

Fig. 3. FGM/Cluster data on 19 December 2001, 02:00-04:00 UT. Average power spectral density of the compressive magnetic fluctuations (upper panel) and of the transverse fluctuations (lower panel). In each panel, the solid line is the average spectrum for large $\Theta_{B V}$ angles, and the dashed line for small $\Theta_{B V}$. The vertical dotted line gives the average $f_{c i}$, the diamonds indicate $k c / \omega_{p i}=0.01,0.1$ and 1 .

As we have just seen from Fig. 2 (and as discussed in Sects. 1 and 2.2), the comparison of the energy level of the turbulent fluctuations for different $\Theta_{B V}$ at a given frequency gives us a good estimate of the wave vector anisotropy. We compare now the PSD of the fluctuations observed for large $\Theta_{B V}$ and for small $\Theta_{B V}$ in the whole frequency range, to estimate the wave vector anisotropy in a large domain of scales.

The upper panel of Fig. 3 displays the spectra of the compressive fluctuations $\delta B_{\| l a}^{2}$ for the $10 \%$ of the points of the sample with the largest $\Theta_{B V}$ (la= large angles, solid line), and $\delta B_{\| s a}^{2}$ for the $10 \%$ of the points with the smallest $\Theta_{B V}$ ( $s a=$ small angles, dashed line). At frequencies below $0.06 \mathrm{~Hz}\left(k c / \omega_{p i}=0.1\right.$, indicated by a vertical solid line) the spectra $\delta B_{\| s a}^{2} \simeq \delta B_{\| l a}^{2}$. At higher frequencies, $f>0.06 \mathrm{~Hz}$ (i.e., at smaller scales, $k c / \omega_{p i}>0.1$ ) we observe $\delta B_{\| l a}^{2}>\delta B_{\| s a}^{2}$. This indicates that 2-D turbulence dominates at such small scales. Close to $10 \mathrm{~Hz}$, i.e. at the vicinity of the FGM Nyquist frequency, we see that 

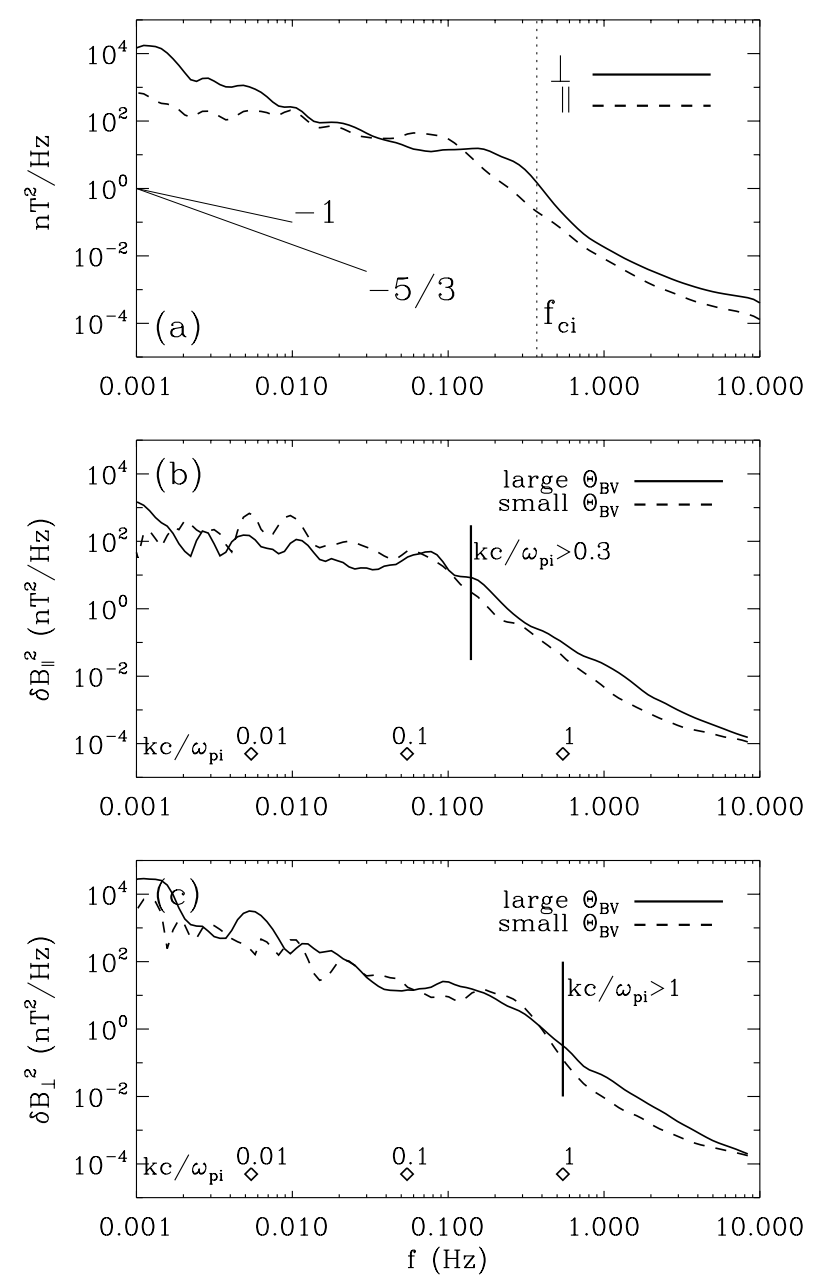

Fig. 4. FGM/Cluster data on 17 May 2002, 08:30-09:30 UT. (a) Average PSD of $\delta B_{\perp}$ ( solid line), PSD of $\delta B_{\|}$(dashed line), the vertical dotted line gives the average $f_{c i}$; (b) the average spectrum of $\delta B_{\|}$for large angles $\Theta_{B V}$ (solid line) and for small angles (dashed line), diamonds indicate $k c / \omega_{p i}=0.01,0.1$ and 1 $\left(k r_{g i}=k c / \omega_{p i}\right.$ in this case); (c) same as (b), for $\delta B_{\perp}$.

$\delta B_{\| l a}^{2} \simeq \delta B_{\| s a}^{2} \simeq 10^{-4} \mathrm{nT}^{2} / \mathrm{Hz}$, that is the sensitivity limit of the FGM instrument. Therefore, the observations at $f>5 \mathrm{~Hz}$ are not physically reliable.

The lower panel of Fig. 3 displays the spectra for the transverse fluctuations for large and small angles $\Theta_{B V}, \delta B_{\perp l a}^{2}$ (solid line) and $\delta B_{\perp s a}^{2}$ (dashed line). We observe that $\delta B_{\perp l a}^{2}$ becomes larger than $\delta B_{\perp s a}^{2}$ at about the same scale of $k c / \omega_{p i} \simeq 0.1$ as for compressive fluctuations. However, here within the spectral bump range, $\sim[0.1,0.3] \mathrm{Hz}$, we observe $\delta B_{\perp l a}^{2} \simeq \delta B_{\perp s a}^{2}$. This is consistent with our previous results that in this short frequency range the 2-D turbulence model is not valid (cf. Fig. 2). A clear dominance of $\delta B_{\perp l a}^{2}$ over $\delta B_{\perp s a}^{2}$ is then observed for $f>0.3 \mathrm{~Hz}\left(k c / \omega_{p i}>0.8\right.$, see a vertical solid line).
These observations allow to conclude that, for $\beta_{i} \simeq 1$, there is a change in the nature of the wave-vector distribution of the magnetic fluctuations in the magnetosheath, in the vicinity of ion characteristic scale: if at MHD scales there is no clear evidence for a dominance of a slab or 2-D geometry of the fluctuations, at ion scales $\left(k c / \omega_{p i}>0.1\right)$ the 2-D turbulence dominates. This is valid for both the Alfvénic and compressive fluctuations. The large scale limit of the 2-D turbulence is, however, different for Alfvénic and compressive fluctuations, and seems to depend on the presence of spectral features, as peaks or bumps. We analyse this point more in details by considering other cases.

\subsection{Other case studies}

The comparison between the spectra for large $\Theta_{B V}$ and for small $\Theta_{B V}$ has been made during four other one-hour intervals, with different average $\beta_{i}$ and different average shock angles $\theta_{B N}$. For the same intervals, Samsonov et al. (2007) display the observed proton temperature anisotropy and the corresponding thresholds for AIC and mirror instabilities.

Figure 4 gives the results of the analysis for an interval (17 May 2002, 08:30-09:30 UT) for which $\theta_{B N} \simeq 70^{\circ}$, $\beta_{i}=(1.6 \pm 0.3), \quad B=(24 \pm 3) \mathrm{nT}, \quad V=(190 \pm 10) \mathrm{km} / \mathrm{s}$, $f_{c i}=(0.37 \pm 0.04) \mathrm{Hz}, \quad c / \omega_{p i}=(55 \pm 2) \mathrm{km} \quad$ and $r_{g i}=(50 \pm 5) \mathrm{km}$.

Figure 4 a gives the average PSD of transverse (solid line) and compressive fluctuations (dashed line). There is a spectral bump for the transverse fluctuations around $0.2 \mathrm{~Hz}$. Below the spectral bump, $\delta B_{\perp}^{2}(f) \sim f^{-5 / 3}$. For the compressive fluctuations there is a spectral bump around $0.07 \mathrm{~Hz}$, probably made of mirror modes. Below the bump, $\delta B_{\|}^{2}(f)$ is close to $f^{-1}$.

In the two other panels of Fig. 4, we display the spectra for large and small $\Theta_{B V}$ for compressive and for transverse fluctuations, respectively. In Fig. $4 \mathrm{~b}$, at frequencies above the bump of $\delta B_{\|}^{2}\left(f>0.1 \mathrm{~Hz}, k c / \omega_{p i} \geq 0.3\right)$ we observe $\delta B_{\| l a}^{2}>\delta B_{\| s a}^{2}$. In Fig. 4c, we observe $\delta B_{\perp l a}^{2} \simeq \delta B_{\perp s a}^{2}$ at large scales (observed at $f<0.05 \mathrm{~Hz}$, i.e. $k c / \omega_{p i}<0.1$ ), but at frequencies above the bump of $\delta B_{\perp}^{2}\left(k c / \omega_{p i}>1\right)$ we observe $\delta B_{\perp l a}^{2}>\delta B_{\perp s a}^{2}$. So, the transverse and compressive fluctuations have $k_{\perp} \gg k_{\|}$at scales smaller than their respective spectral bumps. This confirms the conclusions of Sect. 3.1.

For an interval with $\beta_{i} \simeq 0.8$ and $\theta_{B N} \simeq 70^{\circ}$ (16 December 2001, 08:00-09:00 UT) the average spectrum $\delta B_{\perp}^{2}(f)$ displays a spectral bump around $0.5 \mathrm{~Hz}$. The comparison between the spectra for large and small $\Theta_{B V}$ (not shown) indicates that above the bump, for $k c / \omega_{p i}>1$, the transverse fluctuations can be described by the 2-D-turbulence model. For the compressive fluctuations, this model is valid for a larger range of scales, $k c / \omega_{p i}>0.3$. This confirms the results obtained for $\beta_{i} \simeq 1.6$, shown in Fig. 4 as well as the conclusions of Sect. 3.1. 

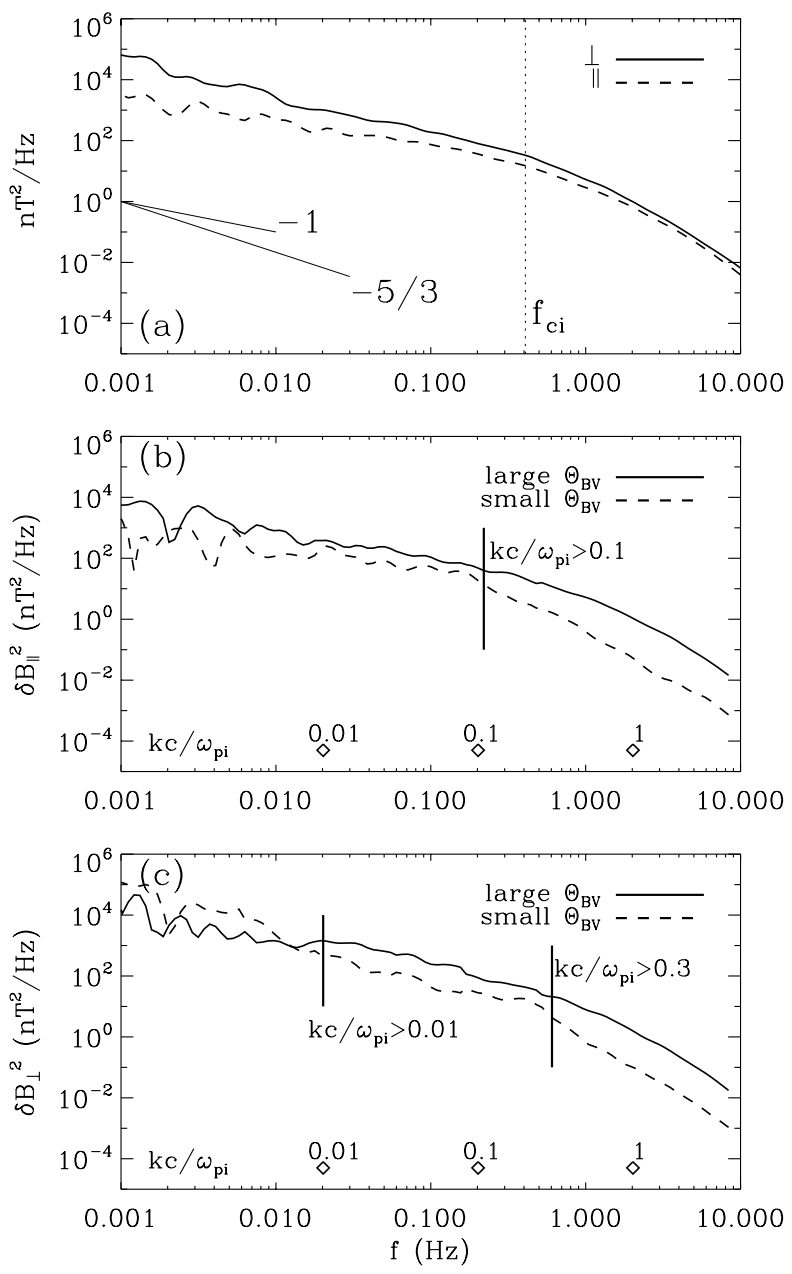

Fig. 5. Same as Fig. 4, but for FGM/Cluster data on 16 December 2001, 05:30-06:30 UT.

In an interval with a larger value of $\beta_{i}$ (17 May 2002, 11:00-12:00 UT, $\left.\beta_{i} \simeq 4.5, \theta_{B N} \simeq 73^{\circ}\right)$, the analysis of the spectra for large and small $\Theta_{B V}$ (not shown) shows that the 2-D turbulence takes place for $k c / \omega_{p i}>0.3$ for the transverse fluctuations, and for $k c / \omega_{p i}>0.2$ for the compressive fluctuations. So, the 2-D turbulence range of scales for the transverse fluctuations is wider in this case.

Figure 5 shows the results of the analysis for an interval (16 December 2001, 05:30-06:30 UT) downstream of an oblique bow shock $\left(\theta_{B N} \simeq 50^{\circ}\right)$, when $\beta_{i}=(10 \pm 3)$, $B=(27 \pm 6) \mathrm{nT}, \quad V=(370 \pm 20) \mathrm{km} / \mathrm{s}, \quad f_{c i}=(0.4 \pm 0.1) \mathrm{Hz}$, $c / \omega_{p i}=(30 \pm 2) \mathrm{km}$ and $r_{g i}=(60 \pm 20) \mathrm{km}$. The wavenumber $k c / \omega_{p i}=1$ appears in the spectrum at $f=(2.0 \pm 0.2) \mathrm{Hz}$ and the wavenumber $k r_{g i}=1$ appears at $f=(1.1 \pm 0.3) \mathrm{Hz}$.

Figure $5 \mathrm{~b}$ shows that at low frequencies (i.e., at large scales, $\left.k c / \omega_{p i}<0.1\right)$ the spectrum for large angle $\delta B_{\| l a}$ dominates slightly at every frequencies. At smaller scales, $k c / \omega_{p i}>0.1$, this dominance is more clear. Figure 5c shows
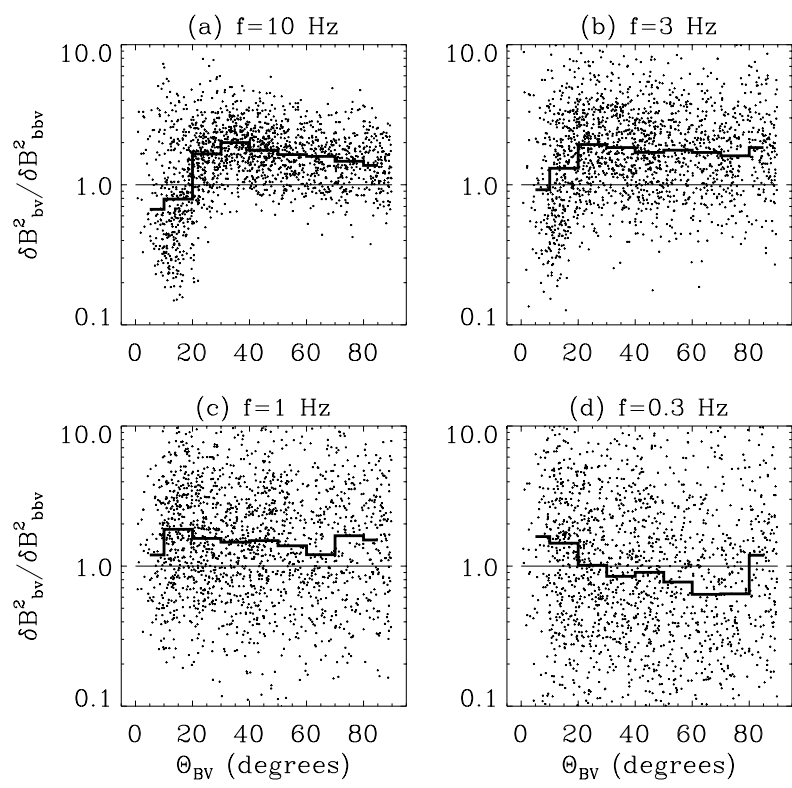

(d) $\mathrm{f}=0.3 \mathrm{~Hz}$

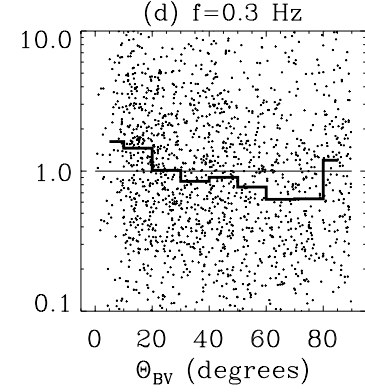

Fig. 6. FGM/Cluster data on 19 December 2001, 02:00-04:00 UT. Scatter plots of the ratio $R=\delta B_{b v}^{2} / \delta B_{b b v}^{2}$ averaged over $4 \mathrm{~s}$, as a function of the angle $\Theta_{B V}$ at (a) $10 \mathrm{~Hz}$, (b) $3 \mathrm{~Hz}$, (c) $1 \mathrm{~Hz}$ and (d) $0.3 \mathrm{~Hz}$. The thick lines give the median value for bins $10^{\circ}$ wide.

that $\delta B_{\perp l a}>\delta B_{\perp s a}$ as far as $k c / \omega_{p i}>0.01$ (wavelengths smaller than $10^{4} \mathrm{~km}$ ), i.e., the $\delta B_{\perp}$ fluctuations can be described by the 2-D-turbulence model at all the scales smaller than the Earth's radius. In this case, with large value of plasma beta, the 2-D turbulence range of scales increases again, but the lower limit of 2-D turbulence is not related to any spectral features, as was observed for smaller $\beta_{i}$.

We may therefore conclude that, at frequencies above the spectral break in the vicinity of $f_{c i}$, the $\delta B_{\perp}$ and $\delta B_{\|}$fluctuations in the magnetosheath have $\mathbf{k}$ mostly perpendicular to $\mathbf{B}$, and this is independent on $\beta_{i}$ and on $\theta_{B N}$. In terms of spatial scales, this is valid for $k c / \omega_{p i}>0.1$ (or $>1$ when spectral features appear in the vicinity of $\left.k c / \omega_{p i}=1\right)$. For high values of $\beta_{i}$, the range of scales of the 2-D turbulence seems to increase: for $\beta_{i} \sim 10$ the fluctuations have $k_{\perp} \gg k_{\|}$ for $k c / \omega_{p i}>0.01$. This small scale spectral anisotropy is also independent on the presence of transverse and/or compressive spectral features (peaks) at larger scales. Nevertheless, for the moderate values of beta $\left(\beta_{i}<3\right)$, these spectral peaks appear as the lower limit of 2-D turbulence.

\section{Gyrotropy of the magnetic fluctuations}

In this section we analyse the anisotropy of the amplitudes of magnetic fluctuations in the plane perpendicular to $\mathbf{B}$. For this purpose, we use the coordinate frame based on $\mathbf{B}$ and $\mathbf{V}$, $(\mathbf{b}, \mathbf{b v}, \mathbf{b b v})$, as explained in Sect. 2.3. 
For the same time interval as Fig. 1, Fig. 6 displays the ratio $R=\delta B_{b v}^{2} / \delta B_{b b v}^{2}$, amplitude of the fluctuations along $\mathbf{B} \times \mathbf{V}$ over the amplitude along $\mathbf{B} \times(\mathbf{B} \times \mathbf{V})$, in the plane perpendicular to $\mathbf{B}$, at four fixed frequencies, as a function of $\Theta_{B V}$.

In Fig. 6a $\left(f=10 \mathrm{~Hz}, k c / \omega_{p i}=23\right)$ and Fig. $6 \mathrm{~b}(f=3 \mathrm{~Hz}$, $\left.k c / \omega_{p i}=7\right)$ the ratio $R$ is larger than 1 for $\Theta_{B V} \gtrsim 20^{\circ}$. The median value decreases and reaches 1 or less for $\Theta_{B V}<20^{\circ}$. A similar dependence was observed by Bieber et al. (1996) and Saur and Bieber (1999) at MHD scales in the solar wind, indicating the dominance of the 2-D turbulence.

At larger scales (Fig. 6c, $f=1 \mathrm{~Hz}, k c / \omega_{p i}=2$ ), in spite of the strong dispersion of $R$, the median values are slightly larger than 1 for any $\Theta_{B V}$ : the 2-D turbulence still dominates. At an even larger scale, the scale of the spectral break (Fig. $6 \mathrm{~d}, f=0.3 \mathrm{~Hz}, k c / \omega_{p i}=0.7$ ), the ratio $R$ is strongly dispersed. The variation of the median does not correspond to a slab or 2-D turbulence. That is in agreement with the results obtained within the spectral break frequency range in Sect. 3.1 (cf. Fig. 2, lowest panel for $\delta B_{\perp}$ ).

The anisotropy of the magnetic fluctuations for the $\left[10^{-3}, 10\right] \mathrm{Hz}$ frequency range is shown in Fig. 7 with average spectra in the three directions $\mathbf{b}$ (dashed line), bv (solid line) and bbv (dotted line). The panels (a) to (f) correspond to increasing values of $\beta_{i}$ for the six considered intervals. For each interval, the vertical solid bar indicates the scale $k c / \omega_{p i}=1$, the dashed-dotted bar indicates $k r_{g i}=1$ and the dotted bar shows $f_{c i}$.

In Fig. $7 \mathrm{a}, \mathrm{b}$ and $\mathrm{c}$, for $k c / \omega_{p i} \geq 1$ we observe that the spectra of the components along $\mathbf{b}$ and along $\mathbf{b b v}$ are nearly equal, $\delta B_{b}^{2} \simeq \delta B_{b b v}^{2}$. In Fig. 7e and $\mathrm{f}, \delta B_{b}^{2}$ is larger than $\delta B_{b v}^{2}$ : the fluctuations are more compressive for the largest values of $\beta_{i}$. All the panels of Fig. 7 show that $\delta B_{b v}^{2}>\delta B_{b b v}^{2}$ for $k c / \omega_{p i} \geq 1\left(k r_{g i} \geq 1\right)$. So, within the 2-D turbulence range the PSD is not gyrotropic at a given frequency.

As we have mentioned in Sect. 1, the observed nongyrotropy in the spacecraft frame can be due to the Doppler shift. Indeed, we have shown in Sect. 3 that the wave vectors $\mathbf{k}$ are mainly perpendicular to $\mathbf{B}$, i.e. $\mathbf{k}$ lies in plane spanned by $\mathbf{b v}$ and bbv. Assuming plane 2-D turbulence, the relation $\mathbf{k}_{\perp} \cdot \delta \mathbf{B}=0$ holds and thus, the wave vectors along bbv (i.e., along the direction of the flow in the plane perpendicular to $\mathbf{B}$, we denote such wave vectors $\mathbf{k}_{\mathbf{b b v}}$ ) contribute to the PSD of $\delta B_{b v}$ and the wave vectors along $\mathbf{b v}\left(\mathbf{k}_{\mathbf{b v}}\right)$ contribute to the PSD of $\delta B_{b b v}$. Even if $I(\mathbf{k})$ is gyrotropic, the fluctuations $\delta B_{b v}$ with $\mathbf{k}_{\mathbf{b b v}}$ suffer a Doppler shift stronger than the fluctuations $\delta B_{b b v}$ with $\mathbf{k}_{\mathbf{b v}}$. For 2-D turbulence, this Doppler shift effect is more pronounced when $\Theta_{B V}$ reaches $90^{\circ}$.

This implies that, for a gyrotropic energy distribution, $I\left(k_{b v}\right) \simeq I\left(k_{b b v}\right)$, in the plane perpendicular to $\mathbf{B}$, and if the energy decreases with $k$, for example as a power law $I(k) \sim k^{-s}$, the observed frequency spectrum $\delta B_{b v}^{2}(f)$ will be more intense than $\delta B_{b b v}^{2}(f)$. In other words, at the same frequency $f$ in the spacecraft frame, we observe the fluctuations with $\left|\mathbf{k}_{\mathbf{b v}}\right|>\left|\mathbf{k}_{\mathbf{b b v}}\right|$. As the larger wave numbers correspond to a weaker intensity (for a monotone energy decrease with $k), \delta B_{b b v}^{2}$ will be smaller than $\delta B_{b v}^{2}$.

Therefore, the non-gyrotropy of $\delta B^{2}(f)$, observed here, could be due to the non-gyrotropy of the Doppler shift, and could be compatible with a gyrotropic distribution of $I(\mathbf{k})$. This is confirmed by the upper panels of Fig. 6: as far as $\mathbf{k}$ is mainly perpendicular to $\mathbf{B}$, the Doppler shift is small and gyrotropic for small $\Theta_{B V}$ and we observe $R \sim 1$, i.e. the PSD is gyrotropic; but for large $\Theta_{B V}, R>1$.

On the other hand, the ratio $R(f)>1$, observed in Fig. 6a and $\mathrm{b}$ for $\Theta_{B V} \simeq 90^{\circ}$, is also compatible with the nongyrotropic k-distribution observed by Sahraoui et al. (2006) near the magnetopause, for $\Theta_{B V} \simeq 90^{\circ}$. In this case study, the authors show that the turbulent cascade develops along $\mathbf{V}$, perpendicular to $\mathbf{B}$ and $\mathbf{n}$, where $\mathbf{n}$ is the normal to the magnetopause. In this geometry, the direction $\mathbf{V}$ is close to bbv. Therefore, a non-gyrotropic $I(\mathbf{k})$ distribution with $\delta B_{b v}^{2}(k) / \delta B_{b b v}^{2}(k)>1$ is expected in the k-domain. This non-gyrotropy of wave vectors is then reinforced by the Doppler shift, and would give $\delta B_{b v}^{2}(f) / \delta B_{b b v}^{2}(f)>1$ in the $f$-domain.

\section{Spectral shapes}

We have mentionned in Sect. 3 that, at frequencies $f<f_{c i}$, below the spectral break, the spectra of the transverse fluctuations $\delta B_{\perp}^{2}(f)$ follow a power law close to $f^{-5 / 3}$ (see Figs. 1 and 4 a). For the six intervals of Fig. 7, Fig. 8 displays compensated plots of the transverse spectra $f^{5 / 3} \delta B_{\perp}^{2}(f)$ (solid lines). On the low frequency side of these plots, we see that the compensated spectra oscillate around a horizontal line, in a frequency range which varies slightly from day to day: a power law $f^{-5 / 3}$ is thus a good approximation for the observations in this frequency range. In Fig. 8a to e, for which $\beta_{i}$ is between 0.8 and 4.5 , the Kolmogoroff $f^{-5 / 3}$ power law is observed below 0.06 or $0.1 \mathrm{~Hz}$, corresponding to scales $k c / \omega_{p i}<0.1$ (see the vertical solid bar).

In Fig. 8 f, for $\beta_{i} \simeq(10 \pm 3)$, the $f^{-5 / 3}$ power law is only observed below $0.01 \mathrm{~Hz}$, i.e., below the 2-D turbulence range of $\delta B_{\perp}$ (see Fig. 5c). Above this frequency, as we see in Fig. $7 \mathrm{f}$, the spectra of all the components are close to an $f^{-1}$ power law and the three spectra have nearly the same intensity, $\delta B_{b}^{2}(f) \simeq \delta B_{b v}^{2}(f) \simeq \delta B_{b b v}^{2}(f)$. This isotropy of the amplitudes of the turbulent fluctuations is natural to observe in a high beta plasma, where the mean magnetic field does not play any important role. Within this frequency range the spectrum can be also formed by a superposition of AIC and mirror waves. For such high $\beta_{i}$, the mirror modes are more unstable than the AIC waves, but they have an important Alfvénic component (Génot et al., 2001), that can also contribute to make fluctuations more isotropic.

Above $f_{c i}$, the spectra $\delta B_{\|}^{2}(f)$ and $\delta B_{\perp}^{2}(f)$ follow similar power laws, see Fig. 7. The compensated spectra $f^{s} \delta B_{\perp}^{2}(f)$ with $s$ between 2 and 3 are presented in Fig. 8 by dashed 

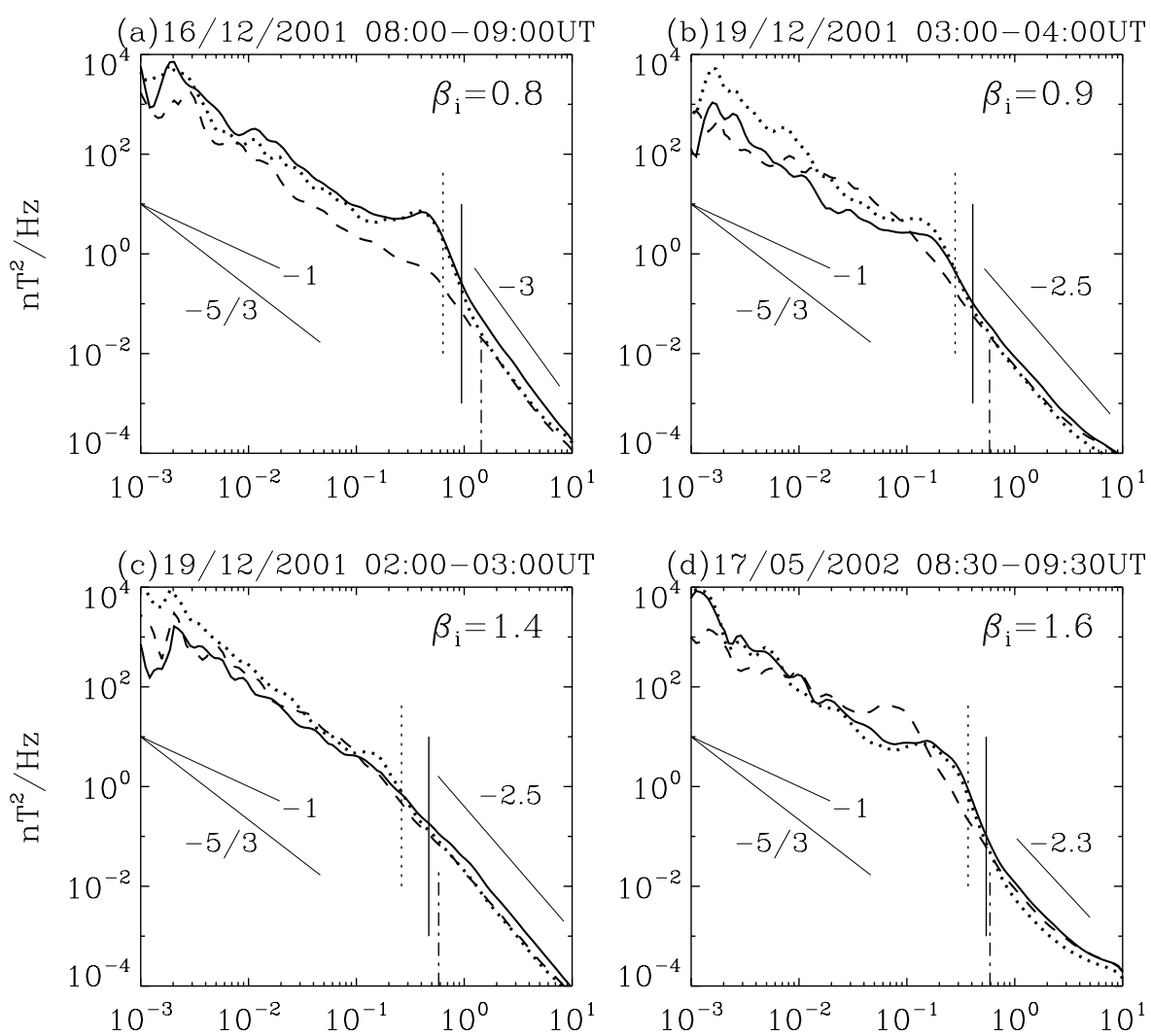

(d) $17 / 05 / 200208: 30-09: 30 U T$

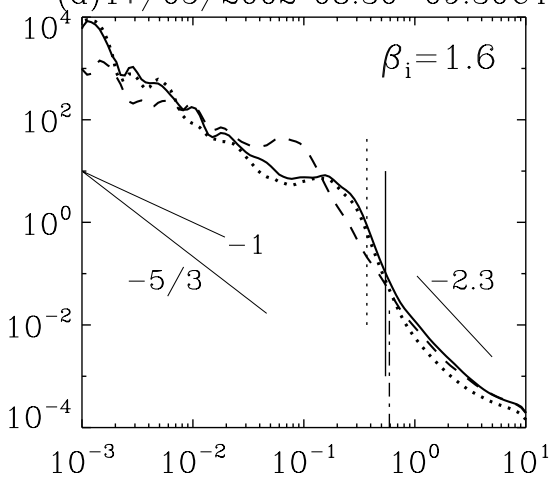

(e)17/05/2002 11:00-12:00UT
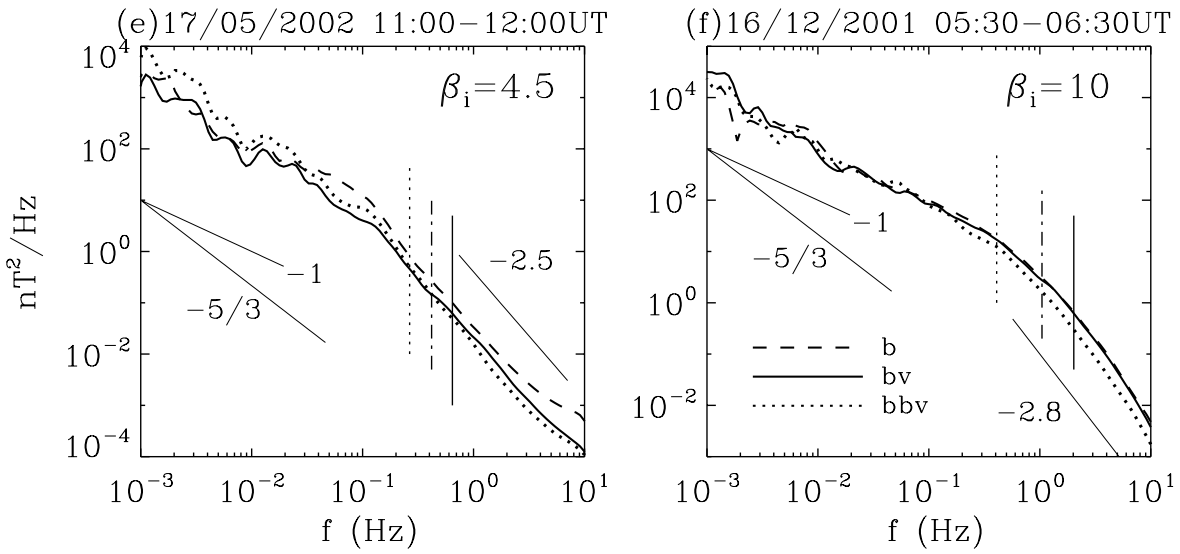

Fig. 7. Average spectra of the magnetic fluctuations in the (b, bv, bbv)-frame, $\mathbf{b}$ is parallel to the $\mathbf{B}$ field (dashed line), bv is parallel to $\mathbf{B} \times \mathbf{V}$ (solid line), $\mathbf{b b v}$ is parallel to $\mathbf{B} \times\left(\mathbf{B} \times \mathbf{V}\right.$ ) (dotted line). For each of the 6 considered one-hour intervals a vertical dotted bar gives $f_{c i}$, a vertical solid bar gives the Doppler shifted wavenumber $k c / \omega_{p i}=1$ and a dashed-dotted bar gives $k r_{g i}=1$. In each panel the shapes of the power laws $f^{-5 / 3}, f^{-1}$ are indicated; in the high frequency range we show the $f^{-s}$ spectral shape, with $s$ determined in Sect. 5, see Fig. 8 .

lines. We see that the compensated spectra $f^{2.5} \delta B_{\perp}^{2}(f)$ oscillate around a horizontal line in a few cases (Fig. $7 \mathrm{~b}, \mathrm{c}$ and e), for different values of $\beta_{i}$. In Fig. 8a, the power law is steeper, $s=3$. Actually, in this case the spectral bump is the most clearly pronounced of the six analyzed intervals. This bump is a signature of the Alfvén vortices, which have their own spectrum $\sim k^{-4}$ or $k^{-6}$, depending on the vortex topol- ogy (Alexandrova, 2008). The superposition of the background turbulence with the coherent structures, like magnetic vortices, can produce the observed steep spectrum. In Fig. 8d we do not observe any clear evidence for a power law spectrum in this frequency range. 

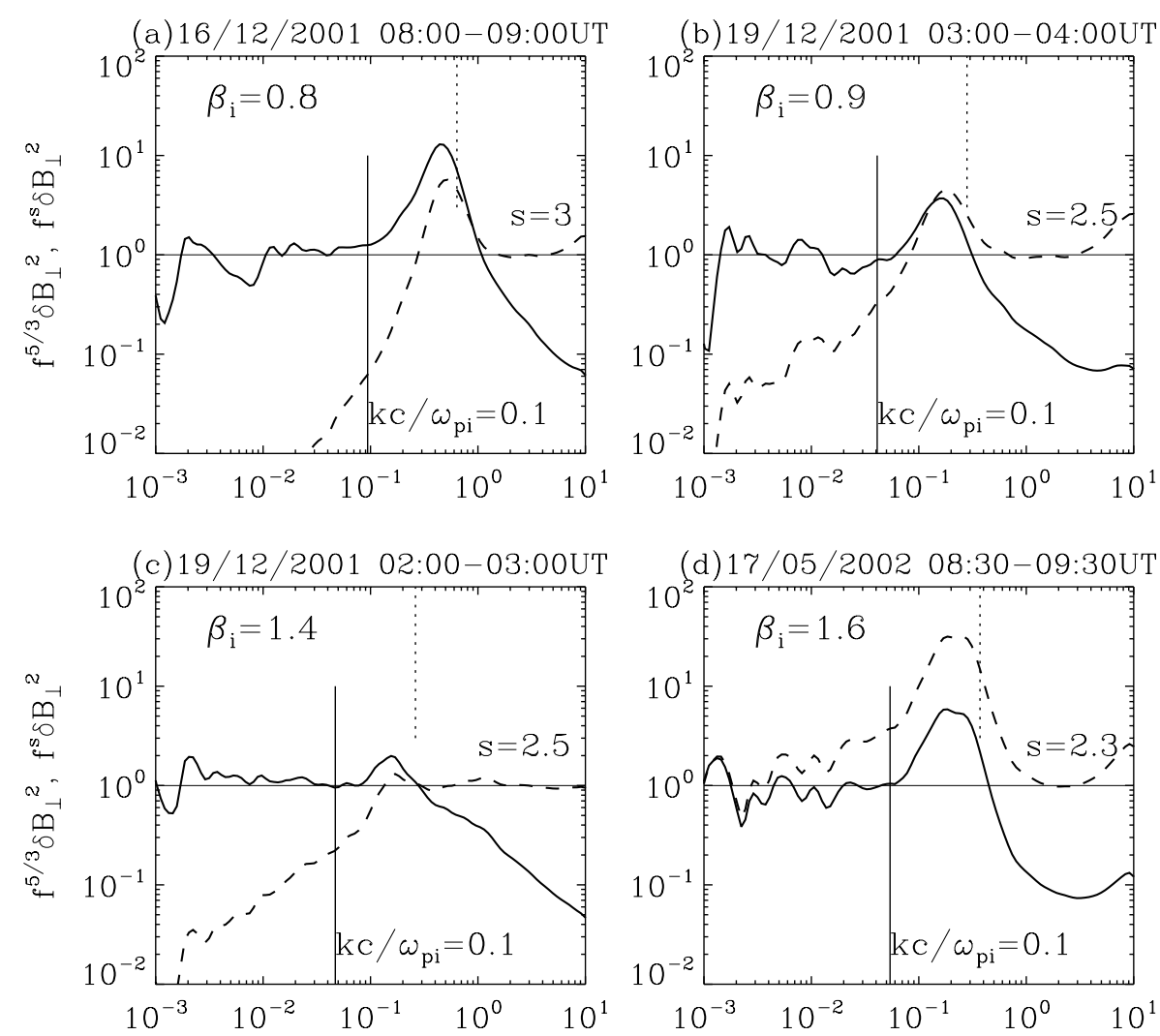

(d) $17 / 05 / 200208: 30-09: 30 U T$
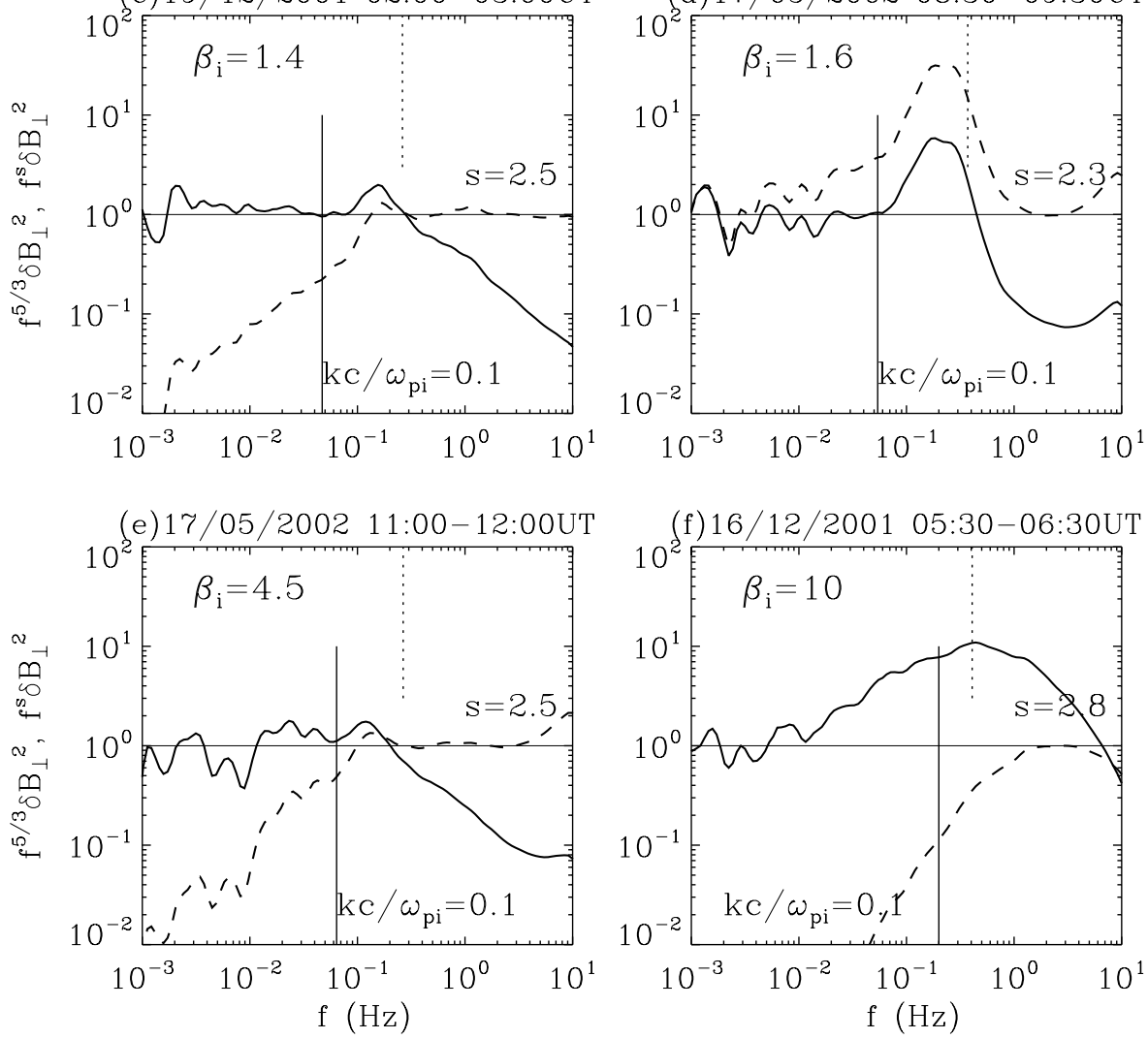

Fig. 8. Compensated spectra $f^{5 / 3} \delta B_{\perp}^{2}(f)$ (solid lines) and $f^{s} \delta B_{\perp}^{2}(f)$ with $s$ indicated in each panel (dashed lines) for the same time periods as Fig. 7.

\section{Summary and discussion}

In the present paper, we have analysed six one-hour intervals in the middle of the terrestrial magnetosheath (at more than one hour from the crossing of the bow shock or of the magnetopause). Precisely, we considered intervals in the magnetosheath flanks: the local times for the three considered days are respectively 08:00, 17:00 and 18:00 h (Lacombe et al., 2006). The ion beta varies from one interval to another, $\beta_{i} \in[0.8,10]$, that allows us to study the plasma turbulence in a very large range of plasma conditions.

\subsection{Spectral shape}

The spectral shape of the magnetic fluctuations in the magnetosheath has been studied by several authors (see Alexandrova, 2008). Rezeau et al. (1999) find a power law $f^{-3.4}$ above $f_{c i}$ in an interval close to the magnetopause. For intervals close to the bow shock, Czaykowska et al. (2001) find 
power laws around $f^{-1}$ below $f_{c i}$, and $f^{-2.6}$ above $f_{c i}$. But in these studies, intervals of $4 \mathrm{~min}$ have been analyzed, so the minimal resolved frequency is about $10^{-2} \mathrm{~Hz}$. In the present paper, the length of the intervals allows to reach frequencies smaller than $10^{-3} \mathrm{~Hz}$.

Here, we present, for the first time, the observations of a Kolmogorov-like inertial range for Alfvénic fluctuations $\delta B_{\perp}^{2}(f) \sim f^{-5 / 3}$ in the frequency range $f<f_{c i}$. It is clearly observed in five of the six studied intervals, those for which $\beta_{i}<5$ and when Alfvénic fluctuations were dominant. Such a Kolmogorov power law is observed in the Alfvénic inertial range of the solar wind turbulence, below the spectral break in the vicinity of $f_{c i}$. The presence of such power law in the magnetosheath flanks is consistent with the estimations made by Alexandrova (2008): in the flanks, the transit time of the plasma is longer than in the subsolar regions, and it is much longer than the time of nonlinear interactions; therefore, the turbulence has enough time to become developed.

In the high frequency range, $f>f_{c i}$, we generally observe $\delta B_{\perp}^{2}(f)$ and $\delta B_{\|}^{2}(f)$ following similar power law $f^{-s}$ with a spectral index $s$ between 2 and 3, in agreement with previous studies.

\subsection{Wave-vector anisotropy}

We analysed here the anisotropy of wave-vector distribution of the magnetic fluctuations from $10^{-3}$ to $10 \mathrm{~Hz}$. This frequency range corresponds to the spatial scales going from $\sim 10$ to $10^{5} \mathrm{~km}$ (from electron to MHD scales). For this analysis we used a statistical method, based on the dependence of the observed magnetic energy at a given frequency on the Doppler shift for different wave vectors (Bieber et al., 1996; Horbury et al., 2005; Mangeney et al., 2006).

Within the inertial range of the magnetosheath turbulence $\left(f<f_{c i}, k c / \omega_{p i}<1, k r_{g i}<1\right)$, we do not observe a clear evidence of wave-vector anisotropy. It can be related to the fact that linearly unstable modes, such as AIC modes with $\mathbf{k}$ mainly parallel to $\mathbf{B}$ and mirror modes with $\mathbf{k}$ mainly perpendicular to $\mathbf{B}$, together with Alfvén vortices with $k_{\perp} \gg k_{\|}$ co-exist in this frequency range.

However, above the spectral break in the vicinity of the ion characteristic scales $\left(f>f_{c i}, k c / \omega_{p i}>1, k r_{g i}>1\right.$ and up to electron scales), we observe a clear evidence of 2-D turbulence with $k_{\perp} \gg k_{\|}$for both $\delta B_{\perp}$ and $\delta B_{\|}$, and independently on $\beta_{i}$, on the bow-shock geometry $\theta_{B N}$, and on the wave activity within the inertial range at larger scales. This wave vector anisotropy seems to be a general property of the small scale turbulence in the Earth's magnetosheath.

The range of wavenumbers of this 2-D turbulence sometimes goes down to $k c / \omega_{p i} \simeq 0.1$ (or even to $k c / \omega_{p i} \simeq 0.01$ ), but usually it is limited by $k c / \omega_{p i} \simeq 1$, while at $k c / \omega_{p i}<1$ spectral features (peaks or bumps) appear. As we can conclude from the work of Mangeney et al. (2006), the largest wavenumbers of the 2-D turbulence are observed around $k c / \omega_{p i} \sim 100$, where the dissipation of electromagnetic tur- bulence begins. This last conjecture must be verified by a deeper analysis.

\subsection{Anisotropy of magnetic fluctuations}

Analyzing the anisotropy of the amplitudes of turbulent fluctuations, we usually find that $\delta B_{\perp}^{2}>\delta B_{\|}^{2}$; but for the largest plasma $\beta_{i}$, the fluctuations are more isotropic $\delta B_{\perp}^{2} \sim \delta B_{\|}^{2}$. This is valid for both the large scale inertial range and the small scale 2-D turbulence. The dominance of $\delta B_{\|}^{2}$ happens only locally in the turbulent spectrum, indicating the presence of an unstable mirror mode.

Concerning the gyrotropy of the amplitude of the magnetic fluctuations in the plane perpendicular to $\mathbf{B}$, there is no universal behavior at large scales. At smaller scales, within the frequency range $[0.3-10] \mathrm{Hz}$ and for any $\beta_{i}$, the 2-D turbulence is observed to be non-gyrotropic: the energy $\delta B_{b v}^{2}$ along the direction perpendicular to $\mathbf{V}$ and $\mathbf{B}$ is larger than the energy $\delta B_{b b v}^{2}$ along the projection of $\mathbf{V}$ in the plane perpendicular to $\mathbf{B}$. This non-gyrotropy might be a consequence of different Doppler shifts for fluctuations with $\mathbf{k}$ parallel and perpendicular to $\mathbf{V}$ in the plane perpendicular to $\mathbf{B}$. The nongyrotropy at a fixed $f$ is compatible with gyrotropic fluctuations at a given $\mathbf{k}$. On the other hand such a non-gyrotropy will be also observed if the k-distribution is not gyrotropic, but is aligned with the plasma flow, as was observed by Sahraoui et al. (2006) in the vicinity of the magnetopause.

Acknowledgements. We thank Jean-Michel Bosqued for providing the CIS/HIA proton data, and Nicole Cornilleau-Wehrlin for the STAFF-SA data. We thank Joachim Saur for constructive comments on the paper. We are very grateful to the team of the Cluster Magnetic field investigation, to the team of the STAFF instrument, and to the Cluster Active Archive (CAA/ESA).

Topical Editor R. Nakamura thanks two anonymous referees for their help in evaluating this paper.

\section{References}

Alexandrova, O.: Solar wind vs magnetosheath turbulence and Alfvén vortices, Nonlin. Processes Geophys., 15, 95-108, 2008, http://www.nonlin-processes-geophys.net/15/95/2008/.

Alexandrova, O., Mangeney, A., Maksimovic, M., CornilleauWehrlin, N., Bosqued, J.-M., and André, M.: Alfvén vortex filaments observed in the magnetosheath downstream of a quasi-perpendicular bow shock, J. Geophys. Res., 111, A12208, doi:10.1029/2006JA011934, 2006.

Alexandrova, O., Mangeney, A., Maksimovic, M., Lacombe, C., Cornilleau-Wehrlin, N., Lucek, E. A., Décréau, P. M. E., Bosqued, J.-M., Travnicek, P., and Fazakerley, A. N.: Cluster observations of finite amplitude Alfvén waves and small-scale magnetic filaments downstream of a quasi-perpendicular shock, J. Geophys. Res., 109, A05207, doi:10.1029/2003JA010056, 2004.

Balogh, A., Carr, C. M., Acuña, M. H., et al.: The Cluster Magnetic Field Investigation: overview of in-flight performance and initial results, Ann. Geophys., 19, 1207-1217, 2001, http://www.ann-geophys.net/19/1207/2001/. 
Bieber, J.W., Wanner, W., and Matthaeus, W. H.: Dominant twodimensional solar wind turbulence with implications for cosmic ray transport, J. Geophys. Res., 101, 2511-2522, 1996.

Constantinescu, O. D., Glassmeier, K.-H., Décréau, P. M. E., Fränz, M., and Fornaçon, K. H.: Low frequency wave source in the outer magnetosphere, magnetosheath, and near Earth solar wind, Ann. Geophys., 25, 2217-2228, 2007, http://www.ann-geophys.net/25/2217/2007/.

Czaykowska, A., Bauer, T. M., Treumann, R. A., and Baumjohann, W.: Magnetic fluctuations across the Earth's bow shock, Ann. Geophys., 19, 275-287, 2001, http://www.ann-geophys.net/19/275/2001/.

Génot, V., Schwartz, S. J., Mazelle, C., Balikhin, M., Dunlop, M., and Bauer, T. M.: Kinetic study of the mirror mode, J. Geophys. Res., 106, 21 611, doi:10.1029/2000JA000457, 2001.

Horbury, T. S., Forman, M. A., and Oughton, S.: Spacecraft observations of solar wind turbulence: an overview, Plasma Phys. Controlled Fusion, 47, B703-B717, 2005.

Lacombe, C., Samsonov, A. A., Mangeney, A., Maksimovic, M., Cornilleau-Wehrlin, N., Harvey, C. C., Bosqued, J.-M., and Trávníček, P.: Cluster observations in the magnetosheath: 2. Intensity of the turbulence at electron scales, Ann. Geophys., 24, 3523-3531, 2006, http://www.ann-geophys.net/24/3523/2006/.

Lucek, E. A., Constantinescu, D., Goldstein, M. L., Pickett, J. S., Pinçon, J.-L., Sahraoui, F., Treumann, R. A., and Walker, S. N.: The Magnetosheath, Space Sci. Rev., 118, 95-152, 2005.

Mangeney, A., Lacombe, C., Maksimovic, M., Samsonov, A. A., Cornilleau-Wehrlin, N., Harvey, C. C., Bosqued, J.-M., and Trávníček, P.: Cluster observations in the magnetosheath: 1. Anisotropy of the wave vector distribution of the turbulence at electron scales, Ann. Geophys., 24, 3507-3521, 2006, http://www.ann-geophys.net/24/3507/2006/.

Narita, Y., Glassmeier, K.-H., Fornaçon, K. H., Richter, I., Schäfer, S., Motschmann, U., Dandouras, I., Rème, H., and Georgescu, E.: Low-frequency wave characteristics in the upstream and downstream regime of the terrestrial bow shock, J. Geophys. Res., 111, A01203, doi:10.1029/2005JA011231, 2006.
Narita, Y. and Glassmeier, K.-H.: Propagation pattern of low frequency waves in the terrestrial magnetosheath, Ann. Geophys., 24, 2441-2444, 2006, http://www.ann-geophys.net/24/2441/2006/.

Rème, Aoustin, H. C., Bosqued, J. M., et al.: First multispacecraft ion measurements in and near the Earth's magnetosphere with the identical Cluster ion spectrometry (CIS) experiment, Ann. Geophys., 19, 1303-1354, 2001, http://www.ann-geophys.net/19/1303/2001/.

Rezeau, L., Belmont, G., Cornilleau-Wehrlin, N., and Reberac, F.: Spectral law and polarization properties of the low frequency waves at the magnetopause, Geophys. Res. Lett., 26, 651-654, 1999.

Sahraoui, F., Belmont, G., Rezeau, L., Cornilleau-Wehrlin, N., Pinçon, J. L., and Balogh, A.: Anisotropic turbulent spectra in the terrestrial magnetosheath as seen by the Cluster spacecraft, Phys. Rev. Lett., 96, 075002, doi:10.1103/PhysRevLett.96.075002, 2006.

Samsonov, A. A., Alexandrova, O., Lacombe, C., Maksimovic, M., and Gary, S. P.: Proton temperature anisotropy in the magnetosheath: comparison of 3-D MHD modelling with Cluster data, Ann. Geophys., 25, 1157-1173, 2007, http://www.ann-geophys.net/25/1157/2007/.

Saur, J. and Bieber, J. W.,: Geometry of low-frequency solar wind magnetic turbulence: Evidence for radially aligned Alfénic fluctuations, J. Geophys. Res., 104, 9975-9988, 1999.

Schäfer, S., Glassmeier, K.-H., Narita, Y., Fornaçon, K. H., Dandouras, I., and Fränz, M.: Statistical phase propagation and dispersion analysis of low frequency waves in the magnetosheath, Ann. Geophys., 23, 3339-3349, 2005, http://www.ann-geophys.net/23/3339/2005/.

Schwartz, S. J., Burgess, D., and Moses, J. J.: Low-frequency waves in the Earth's magnetosheath: present status, Ann. Geophys., 14, 1134-1150, 1996, http://www.ann-geophys.net/14/1134/1996/. 